\title{
BM] Global Health Time-critical conditions: assessment of burden and access to care using verbal autopsy in Agincourt, South Africa
}

\author{
Andrew Fraser (D) , ${ }^{1}$ Jessica Newberry Le Vay, ${ }^{2}$ Peter Byass (D) , $3,4,5$ \\ Stephen Tollman (D) , ${ }^{4}$ Kathleen Kahn (1) , ${ }^{4}$ Lucia D'Ambruoso (1) , ${ }^{4,5}$
} Justine I Davies (1) 4,6

\begin{abstract}
To cite: Fraser $A$
Newberry Le Vay J, Byass P, et al. Time-critical conditions: assessment of burden and access to care using verbal autopsy in Agincourt, South Africa. BMJ Global Health 2020;5:e002289. doi:10.1136/ bmjgh-2020-002289
\end{abstract}

\section{Handling editor Sanni Yaya}

LD and JID are co-senior authors

Received 4 January 2020 Revised 19 March 2020 Accepted 27 March 2020
Check for updates

(c) Author(s) (or their employer(s)) 2020. Re-use permitted under CC BY-NC. No commercial re-use. See rights and permissions. Published by BMJ.

For numbered affiliations see end of article.

Correspondence to Dr Andrew Fraser; andy.fraser@gstt.nhs.uk

\section{ABSTRACT}

Background Time-critical conditions (TCC) are estimated to cause substantial mortality in low and middleincome countries. However, quantification of deaths and identification of contributing factors to those deaths are challenging in settings with poor health records.

Aim To use verbal autopsy (VA) data from the Agincourt health and sociodemographic surveillance system in rural South Africa to quantify the burden of deaths from TCC and to evaluate the barriers in seeking, reaching and receiving quality care for TCC leading to death.

Methodology Deaths from 1993 to 2015 were analysed to identify causality from TCC. Deaths due to TCC were categorised as communicable, non-communicable, maternal, neonatal or injury-related. Proportion of deaths from TCC by age, sex, condition type and temporal trends was described. Deaths due to TCC from 2012 to 2015 were further examined by circumstances of mortality (CoM) indicators embedded in VA. Healthcare access, at illness onset and during the final day of life, as well as place of death, was extracted from free text summaries. Summaries were also analysed qualitatively using a Three Delays framework to identify barriers to healthcare.

Results 0 15305 deaths, 5885 (38.45\%) were due to TCC. Non-communicable diseases were the most prevalent cause of death from TCC (2961/5885 cases, $50.31 \%)$. CoM indicators highlighted delays in a quarter of deaths due to TCC, most frequently in seeking care. The most common pattern of healthcare access was to die outwith a facility, having sought no healthcare (409/1324 cases, $30.89 \%$ ). Issues in receipt of quality care were identified by qualitative analysis.

Conclusion TCCs are responsible for a substantial burden of deaths in this rural South African population. Delays in seeking and receiving quality care were more prominent than those in reaching care, and thus further research and solution development should focus on healthcare-seeking behaviour and quality care provision.

\section{BACKGROUND}

Time-critical conditions (TCC) are a substantial cause of mortality worldwide, responsible for an estimated $45 \%-54 \%$ of all deaths. $^{12}$ These conditions, such as sepsis

\section{Key questions}

What is already known?

- Time-critical conditions (TCC) are estimated to be a substantial cause of mortality in low and middleincome countries, yet there is a lack of consensus regarding the definition of TCC.

- Quantification of deaths and identification of contributing factors to those deaths are challenging in settings with poor medical records.

What are the new findings?

- A locally contextualised definition of TCC was developed.

- There is a substantial burden of deaths due to TCC in this rural South African population.

- Deaths from TCC are mostly due to noncommunicable diseases and have been increasing since 2004 in this population.

- The most significant burden to healthcare in this population for TCC appears to be a delay in seeking care, but quality of care issues were also highlighted by the study. Issues in reaching care were not prominently identified.

\section{What do the new findings imply?}

- Further research and development of solutions for TCC should be focused on improving healthcareseeking behaviour and provision of quality care at facilities.

and myocardial infarction, benefit from highquality emergency care, and rapidly lead to death if left untreated. The burden of deaths due to TCC is much greater in low and middle-income countries (LMICs) than in high-income countries, the difference being around threefold. ${ }^{3}$ Despite the urgent need to respond to this burden, health system provision for acute care has not historically received attention in funding or research, with focus instead on care for specific diseases. ${ }^{45}$ Knowledge of the burden of TCC is inadequate due to a lack of systematic health surveillance of between two-thirds to three-quarters of the 
global population, ${ }^{6}$ with undocumented deaths being common in LMICs. ${ }^{7}$ The lack of a universal definition of TCC adds to difficulty in its study. ${ }^{138-11}$

Building on the World Health Assembly (WHA) Resolution 60.22, which called for improvements in acute care in LMICs, ${ }^{12}$ WHA Resolution 72.16 adds further urgency to the need for quality emergency care in these settings. ${ }^{13}$ The provision of quality acute care is fundamental to achieving improved outcomes for TCC and many health-related Sustainable Development Goals (SDG). ${ }^{14}$ However, a deeper understanding of access to care for TCC is needed to identify gaps and prioritise appropriate prehospital and facility interventions. ${ }^{10}$ Verbal autopsy (VA) is a method to determine cause of death in otherwise unregistered populations ${ }^{15} 16$ by means of a structured interview with a relative or carer of the deceased undertaken by a trained interviewer, within 1 year of death. During the interview, questions aim to ascertain cause of death and circumstances surrounding death. Computer models, such as InterVA-5, are available to interpret large amounts of data to assign causes of death. ${ }^{17}$ VA therefore presents an opportunity to ascertain the burden of deaths due to TCC in settings where health records are lacking.

Ten questions on the circumstances surrounding death, ${ }^{18}$ added to the WHO VA process in 2012, allow establishment of where delays occur in the process of accessing care. ${ }^{19}$ These questions were informed by the Three Delays framework, which structures delays in accessing care into three areas-seeking, reaching and receiving quality care (also known as the first, second and third delays, respectively).$^{20}$ The Three Delays framework was developed to study emergency obstetric care, but has since been used in the study of care for sepsis, ${ }^{21}$ perinatal presentations, ${ }^{22}$ hip fracture ${ }^{23}$ and trauma. ${ }^{19}$ This framework has also been proposed to conceptualise deficiencies in systems of emergency care. ${ }^{24}$

In addition to direct responses to questions, VA interviewers also record information surrounding the death in a free text summary format. This account allows further exploration of access to care and care-seeking behaviours around the time of death, although in a less structured manner.

This study uses VA data to assess mortality, delays in access to care and care-seeking behaviour for TCC in a population residing in a rural area of South Africa.

\section{METHODS}

\section{Study setting}

The study was based at the Rural Public Health and Health Transitions Research Unit of the Medical Research Council and University of the Witwatersrand in Agincourt, South Africa. The Agincourt unit has undertaken health and demographic surveillance surveys (HDSS) since 1992, including conducting annual VA on any deaths reported in the enumerated population. ${ }^{25}$ It is one of three HDSS sites in South Africa comprising the South African Population Research Infrastructure
Network (SAPRIN), as well as being a member of the International Network for the Demographic Evaluation of Populations and Their Health (INDEPTH).

Agincourt is located in a rural area of Mpumalanga, one of the nine provinces of South Africa, in the northeast of the country. Public health services in the country are managed by each province, ${ }^{26}$ with further division into health regions, Agincourt being in the Ehlanzeni region under the jurisdiction of the Mpumalanga Department of Health. ${ }^{27}$ Most interactions with healthcare in South Africa are based at the primary care level, in nurse-led clinics. District hospitals provide the majority of hospitalbased emergency care and are usually staffed by nonspecialist medical officers, with the ability to refer on to regional hospitals for more specialist input. ${ }^{28}$

In Mpumalanga there are 287 primary healthcare facilities, 23 district hospitals, 3 regional and 2 tertiary hospitals. Due to the rural nature of the region, there are also mobile clinics. Ambulance services in the region aim to provide prehospital medical services and interhospital transfers within national targets of 40 minutes in rural areas. ${ }^{27}$ We are not aware of any studies that have assessed whether these targets are met.

\section{Methodologies}

This study employed mixed methodologies using VA data from 1993 to 2015. Data from 2015 were the most recent data available to us at the time of research. Data from 1992 were excluded as the VA process had not been fully established that year.

The literature was scoped for pre-existing definitions of time-criticality and lists of conditions categorised as timecritical that were appropriate to our setting. ${ }^{138-11}$ Those conditions which were defined as 'requiring prompt medical care within twelve hours from the onset of symptoms recognised by a layperson to prevent death' by Hsiao et al were chosen as the basis for our study. ${ }^{10}$ This was due to their definition being used in a large VA project (the Million Death Study), its applicability to an LMIC setting and the availability of the precise list of conditions, using International Classification of Diseases, 10th Revision (ICD-10) codes. Study authors, who had extensive research or clinical expertise in the local area, refined the list and also reviewed causes of death in the VA data set not already classified by Hsiao et al. Consensus on which conditions to define as time-critical was met after discussion between authors.

TCCs defined using ICD-10 were then mapped onto relevant VA codes. Deaths where likelihood of correct cause of death assigned by Inter-VA5 was less than or equal to $50 \%$ were excluded. Deaths were categorised as TCC or not. Numbers and percentages of deaths due to TCC were described according to age group, sex and time period. Deaths from TCC were disaggregated by condition type in the same manner as Hsiao et al: communicable, neonatal, maternal, non-communicable and injury-related. ${ }^{10}$ 
Deaths from TCC were divided into 10-year age groupings. For the age group below 10 years, deaths were also shown for neonates ( $<28$ days), infants ( $\geq 28$ days to $<1$ year) and from 1 to 5 years. Time was divided into 5-year periods, except the last time period (2013-2015), which encompassed 3 years.

The 10 circumstances of mortality (CoM) indicators, as added to the VA process in $2012,{ }^{18}$ were attributed to delays in seeking care (first delay), reaching care (second delay) and receiving quality care (third delay). An additional indicator from the main VA questions regarding patients discharged while still unwell was added to also indicate a delay in receiving quality care. Indicators of delays in seeking care were (1) doubts over the requirements for medical care, (2) use of traditional medicine, (3) lack of use of telephone to seek help, and (4) perceived prohibitive costs. Indicators of delays in reaching care were (1) lack of attendance to a facility, (2) lack of use of motorised transport to reach a facility, and (3) duration of over 2 hours to reach a facility. Indicators of delays in receiving quality care were (1) issues during admission, (2) issues with treatment, (3) issues pertaining to treatment and investigations, and (4) patients discharged while still unwell. As the CoM indicators were only recorded from 2012 and after, only deaths from 2012 onward were considered for this analysis. The number of time-critical deaths which experienced each delay was calculated.

\section{Statistical analysis}

Quantitative analyses were done using SPSS statistics V.24. ${ }^{29}$ Non-parametric data are described as median (IQR). Proportions of deaths due to TCC are shown for age group, sex and 5-year time period. $\chi^{2}$ was used to test the association between TCC and sex; associations between TCC by age and year were tested using nonparametric linear regression, with age and year entered as continuous variables. As an exploratory study, no power calculation was performed.

Free text summaries analysis: quantitative

The free text portion of the VA interview is a summary of the discussion between interviewer and respondent. Notes are taken during the interview and the full narrative is written up by the interviewer following the interview; these contain details of the time leading up to each death that are not possible to capture on the binary responses of the VA questionnaire. These were examined to determine patterns in healthcare access prior to death. Only deaths due to TCC from 2012 to 2015 were analysed, in order to align with the CoM indicator analysis. Information on the following was extracted from the free text summaries: the initial healthcare type accessed (first healthcare act), all healthcare types accessed in the final day of life and the place of death. Cases were excluded if any one of these information points could not be determined. Those dying on the same day as attendance to hospital were classified as accessing the emergency department in the final day of life, whereas those with a hospital stay greater than 1 day prior to death were classified as inpatients.

Free text summaries analysis: qualitative

Free text summaries were further analysed using a qualitative combined inductive and deductive approach. A set of a priori codes related to the first, second and third delays of the Thaddeus and Maine framework were developed. ${ }^{20}$ Free text summaries were coded using NVivo V. $10^{30}$ and an iterative process allowed for re-review with emergent codes, until saturation was reached.

\section{RESULTS}

The list of TCCs and corresponding ICD-10 codes used in this study is presented in the online supplementary table A1. Characteristics of the deceased are shown in table 1. Of the 15305 VAs performed from 1993 to 2015, a total of 5885 deaths were classified as time-critical $(38.45 \%)$. Most time-critical deaths were from non-communicable diseases (NCD; 2961, 50.31\%). Communicable disease (1483, 25.20\%), injury-related (1042, 17.71\%), neonatal (287, 4.87\%) and maternal (112, 1.90\%) deaths made up the remainder.

The most common causes of death for each category were: obstetric haemorrhage $(58.93 \%)$ for the maternal category, indeterminate $(38.50 \%)$ for the NCD category, acute respiratory infection including pneumonia $(81.79 \%)$ for the communicable disease category, neonatal pneumonia $(33.10 \%)$ for the neonatal category and assault $(42.61 \%)$ for the injury-related category. Online supplementary appendix table A2 shows all causes of death identified in the data set, by condition category.

The median age for death due to TCC was 47.54 $(\mathrm{IQR}=42.43)$. The proportion of all deaths which were due to TCC in each age group ranged from $24.32 \%$ (233/958) in the $1-5$ years age group to $74.23 \%$ (288/388) for deaths occurring in the first 28 days of life (table 1 and figure 1; see also online supplementary appendix table A3 for proportion of time-critical deaths attributable to condition category, by age group). The overall pattern was for TCC to increase with increasing age (Spearman's $r$ correlation coefficient of $0.095, p<0.01$ (two tailed)). A slightly smaller proportion of deaths from TCC occurred in females $(2678,45.51 \%)$ than in males $(3207,54.49 \%)$ $\left(\chi^{2}=21.599, \mathrm{p}=<0.001\right)$. The percentage of all deaths due to TCC in 1993 was $42.22 \%$ (133/315) (table 1 and figure 2; see also online supplementary appendix table A4 for proportion of time-critical deaths attributable to condition category, by year). The percentage trended downward, reaching the lowest level in 2004 (221/774, $28.60 \%$ ), then increased thereafter, reaching the highest level in $2014(418 / 842,49.64 \%)$ (Spearman's r correlation coefficient of $0.063, \mathrm{p}<0.01$ (two tailed)).

Of the 3263 all-cause deaths between 2012 and 2015, a total of 1537 were defined as time-critical $(47.10 \%)$. Analysis of the CoM indicators showed that 394 of these 
Table 1 Summary of all-cause deaths and time-critical deaths, according to sex, age group and time period

\begin{tabular}{|c|c|c|c|c|c|c|c|}
\hline & $\begin{array}{l}\text { All deaths } \\
n=15305\end{array}$ & All TCC $n=5885$ & $\begin{array}{l}\text { Maternal } \\
n=112\end{array}$ & NCD n=2961 & CD $n=1483$ & $\begin{array}{l}\text { Neonatal } \\
n=287\end{array}$ & Injury $n=1042$ \\
\hline \multicolumn{8}{|l|}{ Sex } \\
\hline Female & 7328 (47.88\%) & 2678 (45.51\%) & $112(100 \%)$ & 1506 (50.86\%) & 701 (47.27\%) & 133 (46.34\%) & 226 (21.69\%) \\
\hline Male & 7977 (52.12\%) & 3207 (54.49\%) & $0(0 \%)$ & 1455 (49.14\%) & 782 (52.73\%) & 154 (53.66\%) & 816 (78.31\%) \\
\hline \multicolumn{8}{|c|}{ Age group (years) } \\
\hline$<10$ & $2423(15.83 \%)$ & $924(15.70 \%)$ & $0(0 \%)$ & $0(0 \%)$ & $556(37.49 \%)$ & $287(100 \%)$ & $81(7.77 \%)$ \\
\hline Neonate & 388 (2.54\%) & $287(4.88 \%)$ & NA & NA & NA & 287 (100\%) & NA \\
\hline Infant & 908 (5.93\%) & 340 (5.78\%) & $0(0 \%)$ & $0(0 \%)$ & $331(22.32 \%)$ & NA & $9(0.86 \%)$ \\
\hline $1-5$ & 958 (6.26\%) & 233 (3.96\%) & $0(0 \%)$ & $0(0 \%)$ & $192(12.95 \%)$ & NA & 41 (3.93\%) \\
\hline$\geq 10$ to $<20$ & 484 (3.16\%) & 184 (3.13\%) & 10 (8.93\%) & $0(0 \%)$ & 78 (5.26\%) & NA & 96 (9.21\%) \\
\hline$\geq 20$ to $<30$ & 1647 (10.76\%) & 458 (7.78\%) & $32(28.57 \%)$ & $0(0 \%)$ & 137 (9.24\%) & NA & 289 (27.74\%) \\
\hline$\geq 30$ to $<40$ & 2532 (16.54\%) & 837 (14.22\%) & $51(45.54 \%)$ & $401(13.54 \%)$ & $163(10.99 \%)$ & NA & $222(21.31 \%)$ \\
\hline$\geq 40$ to $<50$ & $2051(13.40 \%)$ & $686(11.66 \%)$ & 19 (16.96\%) & $375(12.66 \%)$ & 139 (9.37\%) & NA & $153(14.68 \%)$ \\
\hline$\geq 50$ to $<60$ & $1555(10.16 \%)$ & $622(10.57 \%)$ & $0(0 \%)$ & 444 (14.99\%) & 93 (6.27\%) & NA & $85(8.16 \%)$ \\
\hline$\geq 60$ to $<70$ & 1499 (9.79\%) & $636(10.81 \%)$ & $0(0 \%)$ & 476 (16.08\%) & 106 (7.15\%) & NA & $54(5.18 \%)$ \\
\hline$\geq 70$ & $3114(20.34 \%)$ & $1538(26.13 \%)$ & $0(0 \%)$ & $1265(42.72 \%)$ & $211(14.23 \%)$ & NA & 62 (5.95\%) \\
\hline \multicolumn{8}{|c|}{ Time period (years) } \\
\hline 1993-1997 & $1626(10.62 \%)$ & $682(11.59 \%)$ & $6(5.36 \%)$ & 364 (12.29\%) & $123(8.29 \%)$ & $32(11.15 \%)$ & $157(15.07 \%)$ \\
\hline 1998-2002 & $2644(17.28 \%)$ & $954(16.21 \%)$ & $10(8.93 \%)$ & $492(16.62 \%)$ & $200(13.49 \%)$ & $55(19.16 \%)$ & $197(18.91 \%)$ \\
\hline 2003-2007 & $4209(27.50 \%)$ & $1346(22.87 \%)$ & $22(19.64 \%)$ & $715(24.15 \%)$ & $299(20.16 \%)$ & $74(25.78 \%)$ & $236(22.65 \%)$ \\
\hline 2008-2012 & $4322(28.24 \%)$ & $1700(28.89 \%)$ & $36(32.14 \%)$ & $821(27.73 \%)$ & $508(34.25 \%)$ & $96(33.45 \%)$ & 239 (22.94\%) \\
\hline 2013-2015 & 2504 (16.36\%) & 1203 (20.44\%) & 38 (33.93\%) & $569(19.22 \%)$ & $353(23.80 \%)$ & $30(10.45 \%)$ & $213(20.44 \%)$ \\
\hline
\end{tabular}

Numbers (and percentage) of deaths which are time-critical are also presented for the categories: maternal, non-communicable, communicable, neonatal and injury.

$\mathrm{CD}$, communicable disease; NA, not applicable; NCD, non-communicable disease; TCC, time-critical condition.

(25.63\%) experienced at least one of the three delays and that 647 individual delays were reported. Overall, of the 647 individual delays reported (table 2), $428(66.15 \%)$ were due to a delay in seeking care (first delay), 72 $(11.13 \%)$ due to a delay in reaching care (second delay) and $147(22.72 \%)$ due to a delay in receiving quality care (third delay).

\section{Quantitative free text summaries analysis}

The required data from the free text summaries regarding patterns of healthcare access were available in 1324 of 1537 VAs $(86.14 \%)$ performed between

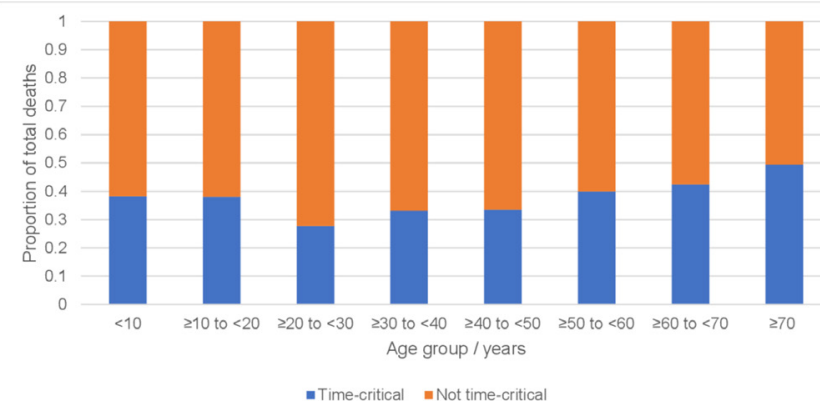

Figure 1 Proportion of total deaths defined as time-critical, by age group (1993-2015).
2012 and 2015. The most common access pattern was to access no healthcare and die outwith a facility (409/1324, 30.89\%). The second most common pattern was to attend hospital and die as an inpatient (218/1324, $16.47 \%)$. In 175 cases $(13.22 \%)$ the pattern was to attend clinic initially, then be admitted to hospital and die as an inpatient. In 73 cases the deceased attended clinic initially, then died without being admitted to a facility $(5.51 \%)$. Other patterns of healthcare access are shown in figure 3.

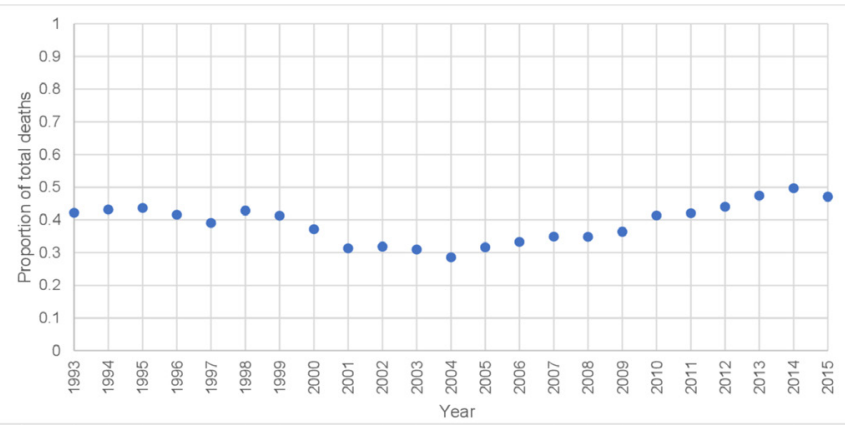

Figure 2 Proportion of total deaths defined as time-critical, over time (1993-2015). 
Table 2 The three delays experienced in deaths from time-critical conditions, as assessed using circumstances of mortality (CoM) indicators

\begin{tabular}{llc}
\hline Delay & CoM indicator & Number of deceased experiencing delay* \\
\hline First delay - seeking care & Doubts about need for care & $39(9.90 \%)$ \\
& Use of telephone & $36(9.14 \%)$ \\
& Use of traditional medicine & $84(21.32 \%)$ \\
& Perceived prohibitive costs & $269(68.27 \%)$ \\
Second delay-reaching care & Travel to health facility & $36(9.14 \%)$ \\
& Use of motorised transport & $36(9.14 \%)$ \\
& Distance (more than 2hours to facility) & $0(0.00 \%)$ \\
Third delay-receiving quality care & Problems with admission & $27(6.85 \%)$ \\
& Problems with treatment & $26(6.60 \%)$ \\
& Problems with medicine & $27(6.85 \%)$ \\
& Discharged while ill & $67(17.01 \%)$ \\
\hline
\end{tabular}

*Percentage does not total $100 \%$ as delays can be experienced by the same deceased person in more than one category. (The denominator is the $\%$ of deceased who responded stating any delay using CoM indicators $(n=394)$.) The total number of delays captured for all deceased was $\mathrm{n}=647$.

\section{Qualitative free text summaries analysis}

Delays in seeking care (first delay)

Interviewers recorded accounts of home-based care, such as the use of left-over or purchased medication

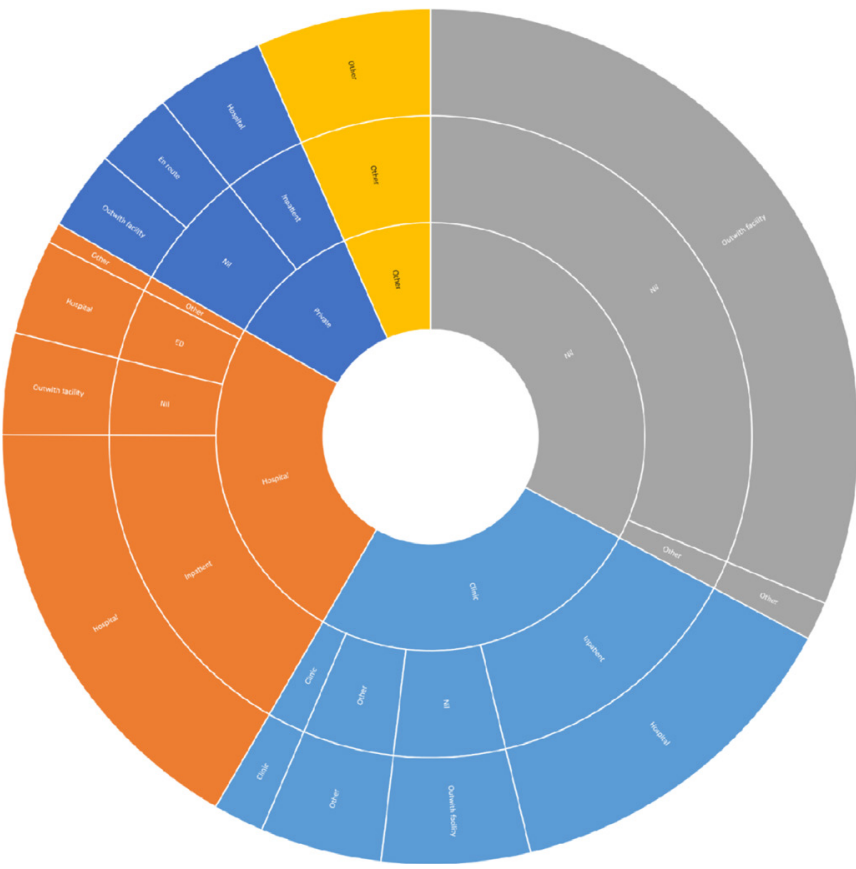

Figure 3 Access to healthcare patterns for time-critical deaths (2012-2015). Inner ring shows first healthcare act, middle ring shows healthcare accessed on final day of life, outer ring shows place of death. Pattern of healthcare access determined by movement from inner to outer ring. Size of wedge proportional to number of cases. Colours attributed according to first healthcare act (grey=nil, light blue=clinic, orange=hospital, dark blue=private, yellow=other). Less common pathways have been amalgamated into 'other' category, which include first healthcare acts of ambulance, already inpatient and traditional healer. ED, emergency department. and home-made oral rehydration solution, commonly in deaths from TCC. Attendance to traditional healers or the church also occurred frequently. Symptoms did not tend to improve after self or traditional treatments, with some dying at home without seeking Western healthcare.

Left over medication was given but nothing changed. [...] They continued giving him left over medication $[\ldots]$ he died at home.

Refusal to attend clinic or hospital was a recurrent theme, but reasons for this were not often recorded. Some cases conveyed negative perceptions of Western medicine:

Nothing was done as they thought Western treatment won't help.

\section{Delays in reaching care (second delay)}

Several of the deceased died en route to a facility using their own transportation, although specific difficulties in obtaining private transportation were not mentioned. Ambulances were not quick enough to prevent death for some:

An ambulance was called but she died at home before the ambulance arrived.

Delays in receiving quality care (third delay)

On referral from clinic to hospital, patients tended to return home prior to arranging transport to hospital, rather than travel directly to hospital by ambulance. Many patients waited until the next day to travel to hospital, with some dying at home. Even when an ambulance was called, some died while waiting for its arrival. Referral to a tertiary or specialist facility from a district hospital could also be problematic.

Treatment was given and referred to hospital. [...] She died the night before she was taken to hospital. 
Doctors tell the family that they will refer her to another hospital [...] she died before they refer her.

Some people died while waiting to be seen or while being attended to at a clinic or emergency department. Clinicians were thought to communicate poorly with patients and relatives, with the likely diagnosis and results of investigations often not being shared.

He was taken to hospital and while waiting on the queue he started to have difficulty in breathing and he died...

X-ray was done again but doctors didn't tell the family what they found.

Patients in clinics and hospitals often did not improve clinically, despite persistence of treatment, and consideration of alternative treatments seemed to be sporadic. Several accounts described clinical assessment and administration of treatment as lacking. Several deaths occurred a short time after attendance to a healthcare facility, while some patients were not well prior to discharge.

She was given water drips, injection and other treatment, nothing has changed [...] She was always given water drips and other treatment. Nothing has changed.

Her sugar level was too high and they fail to control it.

He died at home, the same day of consultation from the clinic.

The free text summaries described a lack of consumables and malfunctioning equipment at clinics and hospitals. Lack of bed space also affected patient care.

Oxygen was not given because it was not working.

Doctor said she was supposed to be admitted but they will not admit her because all the beds were full.

\section{DISCUSSION}

The burden of TCC in this population is substantial, with males and those at extremes of age more likely to die from TCC. The proportion of deaths due to TCC has been rising, accounting for nearly $50 \%$ of all deaths since a nadir in 2004. CoM indicators showed that in around one-quarter of deaths with TCC, any one of the three delays to care was experienced, with the first delay occurring most frequently. Quantitative analysis of the free text summaries indicated the importance of the delays in seeking care, given that around one-third of the reviewed 1324 cases did not seek any care prior to death outwith a facility. On the other hand, qualitative analysis of the free text summaries most frequently described delays in receiving quality care.

The proportion of deaths identified as time-critical is similar to those described by modelled estimates, ${ }^{12}$ as well as a population-level study in India. ${ }^{10}$ That males experienced a slightly greater burden of TCC is likely to be explained by the increased proportion of injury-related deaths in males versus females. This echoes global findings, whereby men account for three-quarters of deaths from road traffic injuries and four-fifths from homicide worldwide, ${ }^{31}$ and also findings from a previous study undertaken in this population. ${ }^{19}$ Deaths in those aged 28 days or less were most likely to be due to a TCC, likely due to the scope of conditions affecting this age group, which tend to be incompatible with life without treatment. The per cent of deaths within an age group which were timecritical remained between $30 \%$ and $40 \%$ until around 70 years, after which it increased; this is likely attributable to the increased NCD burden tending to affect older ages.

The increase in proportion of deaths due to TCC seen from 2004 onward may correspond with the improvements in life expectancy due to progress made in treating the HIV/AIDS epidemic in South Africa. This change would allow those who previously succumbed to HIV/ AIDS to develop TCC. In addition, as a country undergoing both epidemiological and demographic transitions, the burden of NCD, of which there is a substantial time-critical component, will increase. ${ }^{32}$ The increasing risk for some TCC, such as acute cardiovascular disease, likely conspires with under-resourced primary care and primary prevention services to increase the incidence of TCC. ${ }^{33-35}$

Although the CoM indicators showed that only around one-quarter of people who died from TCC experienced any delay in seeking, reaching or receiving quality care, the relative proportions of delays experienced are informative. Delay in seeking care was found to be the most prevalent when using the CoM indicators and this is substantiated by the most common pattern of healthcare access being to access no healthcare and death outwith a facility. The free text summaries provide insight into the drivers for this delay in seeking care. Only a small proportion of cases $(47 / 1324)$ initially accessed traditional medicine. However, of those people who did access traditional medicine, a large proportion died with a traditional healer or outwith a facility, suggesting that accessing traditional healers for the acutely unwell can prevent potentially life-saving access to biomedical care; our qualitative analysis supported this hypothesis. Other groups have shown that education of traditional healers in signposting can improve referral to biomedical care in people with HIV ${ }^{36}$ It would be important to consider whether this would be an efficient use of resources for TCC, given the low numbers that our study identified using this pathway prior to death.

Self-treatment, which usually referred to the administration of left-over medication from a previous healthcare encounter or using home-made oral rehydration solution, was frequently mentioned in the free text summaries as an alternative to formal care in the early stages of the final illness. Although some medications could be appropriate for a particular TCC, the qualitative analysis suggested that these medications were taken without knowledge of the likelihood of its ability to successfully treat that particular condition. The CoM indicators also suggest that no help was sought via the telephone in a relatively small number $(2.34 \%)$ of cases, and the importance of this was 
not detected by the qualitative analysis. In South Africa, there is a single national number specifically for calling an ambulance. ${ }^{37}$ Evidence in other sub-Saharan African countries suggests a poor public awareness of emergency telephone numbers. ${ }^{38}$

The most frequently identified barrier on examining the CoM indicators was costs. Care at public clinics and hospitals is free in South Africa, but there are still direct costs (eg, transport) and indirect costs (eg, loss of wages). These have been shown to affect patients accessing HIV/ AIDS care in South Africa. ${ }^{39}$ Prior personal or community experience of costs for accessing medical care in South Africa may disincline people with TCC from seeking care. This may be a factor in explaining the large proportion of the deceased who did not seek any care in our study population.

That delays in seeking care were quantitatively the most important-using the CoM indicators and the first healthcare act-suggests lack of knowledge of or trust in the ability of a healthcare facility to provide treatment may be an issue in this population. ${ }^{40}$ Indeed, patient refusal to attend a facility was a frequent theme occurring in the free text summaries. Although the reasons for this were not readily identified, a few had views that Western medicine would not work. Previous direct experience or vicarious knowledge of delays in reaching and receiving quality care may have resulted in the decision not to seek care. $^{20}$

Delays in reaching care were only implicated in a small number of cases by the CoM indicators. Few respondents reported the deceased using an ambulance to initially access treatment, and on analysis of the free text, dying outwith a facility (or in clinic) waiting for an ambulance was reported. The patterns of healthcare access showed that a quarter of those initially accessing an ambulance died outside hospital. Prehospital care has been shown to have deficiencies, namely lack of appropriate equipment and inadequate staffing, within other rural areas of South Africa. ${ }^{41}$ Due to lack of a timely ambulance service, or perceptions of poor availability and quality, car tends to be the most common form of motorised transportation used for emergencies in LMICs. ${ }^{38} 42$ In the free text analysis, delays in reaching care appeared greater than on analysis using CoM indicators. Deaths en route to a facility in a small number of cases were revealed but it was not possible to differentiate en route deaths into those using ambulance versus own transport.

Additionally, deaths en route could be argued to be both indicative of a delay in seeking care as well as of reaching care (or the quality of emergency medical services providers) as the illness may have been left too late to be salvageable. Distance from a facility has been found in other studies to be implicated in higher mortality from TCC. ${ }^{43}$ However, distance from a healthcare facility was not a prominent issue in this study, featuring no mention in the qualitative analysis, and none of the deceased were located more than 2 hours from a healthcare facility according to the CoM indicators. This is likely to be accurate given the geographical spread of healthcare facilities and road quality in the Agincourt area. ${ }^{25}$ Geography is only one component of access, which also involves other dimensions, such as awareness and understanding, cultural context and financial constraints. Many studies, which have focused on geographical access to a facility, have found this to be reasonable, however, those considering access using additional dimensions have found this to be lacking. ${ }^{445}$

Despite short distances between facilities, interfacility transfer appeared to be problematic. On referral to hospital from clinic, patients were expected to arrange their own transportation, rather than be transferred by ambulance. Qualitative analysis also showed that transfer to a specialist facility from a district hospital was thought to contribute to mortality. Issues with referral have been shown to be a significant cause of worsened health outcomes in other contexts, such as maternal mortality. ${ }^{46}$ Non-concordance with referral advice by relatives of the deceased has been regarded to be a particular challenge in referring patients from first-level health facilities in similar settings. ${ }^{47}$ Thaddeus and Maine classified issues with referral as a delay in receiving quality care (third delay).$^{20}$ Referral relies on recognition of TCC at referring facilities, and on transportation processes between them; delays due to transportation are usually regarded as a delay in reaching care (second delay). Thus, it could be argued that the act of referring a patient to a higher level facility exposes them to delays in reaching care, having already encountered delays in receiving quality care at the initial facility. The concept of cyclical delays, rather than being simply a linear process, has been discussed in other studies. ${ }^{48}$

A study examining paediatric care in Rwanda identified a key 'fourth delay', characterised as an additional source of delays after leaving a healthcare facility. These occur in the form of lack of follow-up care, difficulty in adherence to treatment plans and abandoning biomedical care for other forms of healthcare, such as traditional healers. ${ }^{49}$ Previous healthcare experiences also impact on this delay.

The second most common pattern of healthcare access for TCG was attendance at hospital and death as an inpatient. Attendance at clinic, then admission to hospital for an inpatient stay, followed by death in hospital was also common. This may either suggest that a substantial proportion of the deceased in this population experienced delays in receiving quality care, or that delays in seeking and reaching care meant that the illness had progressed to be incurable by the time the patient reached the facility. That a large proportion of the deceased were over 70 years old may also contribute to the final illness not being survivable, especially in a population where multimorbidity is prevalent. ${ }^{50}$ However, that these cases managed to overcome the first two delays and attend a facility, suggests that quality of care issues should not be disregarded. 
Poor quality of care at facilities was the most commonly identified issue in the qualitative analysis. Particular areas of concern surrounded communication, assessment of the patient and availability of beds and equipment. However, it must be considered that the free text summaries were written by the interviewer based on the information given to them by the carers of the deceased and may not be a true reflection of experiences in facilities, when relatives may have not been present.

Being discharged while still ill was another quality of care issue identified in the study. On exploration of the conditions associated with this outcome on the CoM indicators, it was found that $31.34 \%(21 / 67)$ of these cases died due to stroke and $23.88 \%(16 / 67)$ of these cases died due to acute respiratory infection (see online supplementary appendix table A5). Terminal cases may have been discharged home to die at the request of the patient, but that could not be determined by this study. The reasoning behind patients being discharged to die at home requires further elucidation, as if facility-based care is perceived as being poor quality by members of the community, it can impact on care-seeking behaviours of subsequent people who require care. ${ }^{20}$

Although the management of TCC is primarily regarded as the provision of quality acute care, primary care and preventative public health strategies play a role in alleviating the burden from TCC. Many TCCs could be prevented through the reduction of risk factors and associated diseases, such as adequate treatment of diabetes mellitus reducing the TCC of diabetic ketoacidosis. ${ }^{51}$ Additionally, primary care providers should be able to recognise TCC and appropriately refer. The focus of VA on events proximate to death does not allow capture of data on primary prevention efforts that could have prevented TCC; further studies of health service availability in the region are needed to provide data regarding this.

Of note are differences in barriers identified by the different methodologies employed in our study. The qualitative review of free text summaries most frequently identified barriers to receiving quality care, echoing the findings of the Lancet Global Health Commission on High Quality Health Systems in the SDG era, which determined that inadequate and poor quality care is common, especially in LMICs. ${ }^{52}$ On the other hand, the CoM indicators showed a prominence of delays in seeking care, as did examination of the patterns of healthcare access. Open and closed questions may have different abilities to detect delays in a given population. A previous study using VA to investigate maternal mortality delays in Burkina Faso and Indonesia found that different delays are reported in open sections of the interview compared with the closed questioning. ${ }^{48}$ This discrepancy could be regarded as a positive feature of using structured and non-structured formats for VA interviews, as it allows a breadth of delays to be captured, with the open, free text sections allowing for expression of cultural perspectives to ascertain issues that are important for health planning.
The mixed methodology of our study identified specific barriers to care in the studied population, which is useful for future health planning. However, the differing results between the quantitative and qualitative aspects make it difficult to draw a conclusion as to whether overall delays in seeking or receiving quality care are more influential in the studied population. That said, using VA in this manner, via the inclusion of analysis of free text summaries, allows for more of the cultural perspective on healthcare access to be understood.

\section{Limitations}

The lack of a universal definition of time-criticality and associated conditions, requiring the need to formulate a locally contextualised classification of TCC using consensus agreement between authors, may limit the direct generalisability of this study, as does the study being conducted in just one area of South Africa. However, the population within the Agincourt HDSS site has characteristics similar to South Africa as a whole. Additionally, the Agincourt HDSS site is part of SAPRIN and the global INDEPTH Network of HDSS sites, allowing the methodologies that we have developed to be applicable more widely.

In terms of time-criticality, Hsiao et al defined timecritical mortality as being from 12 hours from symptom onset to death. ${ }^{10}$ However, temporal certainty is unable to be distinguished using VA data, and also a particular condition may take different amounts of time from symptom onset to death in person to person. The list of conditions was agreed on the principle of time-criticality being likely for most people with these conditions. Survivability of TCC could not be evaluated using this study methodology; thus, although we were able to document where delays were occurring, it was not possible to see if those delays contributed to an untimely death. Some conditions may not be survivable, even with the best medical care available. Edem et al examined a way to define the survivability of a trauma case ${ }^{19}$ but the complexity and plethora of causes for time-critical deaths would limit the transferability of that technique to this study.

The breadth of VA codes meant that our categorisation of TCC may have lacked precision. For example, two ICD-10 codes, such as I10 (essential (primary) hypertension) and I26 (acute pulmonary embolism), defined as not time-critical and time-critical, respectively, were mappable to the same VA code (other and unspecified cardiac disease). This occurred in few instances and, in such cases, the deaths were classified as time-critical. This may have led to an overestimate of TCC, but it is doubtful that these small numbers substantially altered the findings of this analysis.

The pattern of healthcare access was limited in that only three variables were captured. Examining additional stages of healthcare access for these patterns would have given a more detailed and accurate picture. A lack of standardised time period for the free text summaries meant 
that in some cases the described period referred to few hours, whereas in others it could refer to days; however, where possible, only cases were included where the time was reasonably certain.

Blank spaces in the CoM answer field were assumed to denote 'no', but the respondent may not have known the answer. While these limitations may be overcome by combining analysis of CoM with that of the free text, the free text may have only identified certain barriers to care which are perceived to be more important by the respondent, with other delays that still are important to health outcomes not being described.

Quality data from VA are dependent on the accurate recording of an interview with an unbiased interviewee. There may have been a degree of subjectivity in the interviewers when writing the free text summary accounts; additionally, VA is conducted with a carer of the deceased who may not have been present in the facility often enough to reliably comment on the quality of care provided. Recall bias, in that relatives may be more likely to remember a negative healthcare experience than a positive one, may exist. Previous experiences and cultural norms may influence the descriptions of events by relatives of the deceased. VAs also do not provide information about survivors or attributed morbidity.

\section{CONCLUSION}

Our study has found that TCCs are a common and increasing cause of death in this South African population, with the majority being due to NCDs. We have also shown that this population experiences considerable barriers to obtaining quality healthcare for TCC. The most frequently occurring delay shown by the CoM indicators was in seeking care, which was echoed by the most commonly occurring healthcare access pattern being to access no healthcare and die outwith a facility. This suggests that awareness in both the public and healthcare services of the need to access healthcare services is not aligned with the changing burden of conditions. Qualitative review of interviewer free text summaries, however, highlighted the presence of delays in receiving quality care at a facility. LMICs have recognised the need to improve healthcare systems in acute care with WHA Resolution 72.16, which was proposed by Ethiopia and Eswatini. ${ }^{13}$ The methodologies used in this study could be readily be applied to other VA data collected throughout LMICs by HDSS sites and elsewhere. Using recent developments in VA, such as 'Circumstances of Mortality CATegories', could allow for analysis at scale of barriers faced in accessing healthcare. ${ }^{53}$

\section{Author affiliations \\ ${ }^{1}$ Department of Critical Care, Guy's and St Thomas' NHS Foundation Trust, London, UK \\ ${ }^{2}$ Cancer Policy Research Centre, Cancer Research UK, London, UK \\ ${ }^{3}$ Department of Epidemiology and Global Health, Umeå University, Umeå, Sweden}

${ }^{4}$ Medical Research Council/Wits University Rural Public Health and Health Transitions Research Unit (Agincourt), Faculty of Health Sciences, School of Public Health, University of the Witwatersrand, Johannesburg, South Africa

${ }^{5}$ Aberdeen Centre for Health Data Science, Institute of Applied Health Sciences, School of Medicine, Medical Sciences and Dentistry, University of Aberdeen, Aberdeen, UK

${ }^{6}$ Institute of Applied Health Research, University of Birmingham, Birmingham, UK

Acknowledgements We thank Chodziwadziwa Kabudula (Medical Research Council/Wits University Rural Public Health and Health Transitions Research Unit (Agincourt), Faculty of Health Sciences, School of Public Health, University of the Witwatersrand, Johannesburg, South Africa) for his assistance with assembling the Agincourt HDSS data set for our use.

Contributors AF helped develop the idea, performed the analyses, drafted and revised the paper. JNLV helped develop the idea and inputted into the paper drafts. PB refined the data set, inputted into the paper drafts and is responsible for InterVA-5. ST inputted into the paper drafts and is responsible for the Agincourt HDSS. KK inputted into the paper drafts and is responsible for the system of verbal autopsies at Agincourt. LD inputted into the idea, supported the analyses and inputted into the paper drafts. JID led the project, developed the idea, supported the analyses and inputted into the paper drafts and revisions. All authors approved the final paper for submission.

Funding A travel scholarship to enable this project was provided by King's College London. Article processing fees will be covered by University of Birmingham.

Disclaimer These funding sources were not involved in this study design, in the collection, analysis and interpretation of the data, in the writing of the manuscript, nor in the decision to submit the paper for publication.

Competing interests None declared.

Patient and public involvement We did not directly include public involvement in this study. The database, from which this study derives data, is used to inform a public engagement programme at the Medical Research Council/Wits University Rural Public Health and Health Transitions Research Unit (Agincourt).

\section{Patient consent for publication Not required}

Ethics approval Ethical approval for secondary use of the VA data set from MRC/ Wits Agincourt Unit had been granted by the Human Research Ethics Committee (Medical) of the University of the Witwatersrand (M960720 and M110138). Ethical approval was also granted by the Biomedical and Health Sciences, Dentistry, Medicine and Natural and Mathematical Sciences Research Ethics Subcommittees of King's College London (RESCM-17/18-5635).

Provenance and peer review Not commissioned; externally peer reviewed.

Data availability statement Data is not publicly available. Deidentified verbal autopsy data from Medical Research Council/Wits University Rural Public Health and Health Transitions Research Unit (Agincourt) (stephen.tollman@wits.ac.za).

Open access This is an open access article distributed in accordance with the Creative Commons Attribution Non Commercial (CC BY-NC 4.0) license, which permits others to distribute, remix, adapt, build upon this work non-commercially, and license their derivative works on different terms, provided the original work is properly cited, appropriate credit is given, any changes made indicated, and the use is non-commercial. See: http://creativecommons.org/licenses/by-nc/4.0/.

\section{ORCID iDs}

Andrew Fraser http://orcid.org/0000-0002-9203-8692

Peter Byass http://orcid.org/0000-0001-5474-4361

Stephen Tollman http://orcid.org/0000-0003-0744-7588

Kathleen Kahn http://orcid.org/0000-0003-3339-3931

Lucia D'Ambruoso http://orcid.org/0000-0002-8505-3368

Justine I Davies http://orcid.org/0000-0001-6834-1838

\section{REFERENCES}

1 Kobusingye OC, Hyder AA, Bishai D, et al. Emergency medical services. Disease control priorities in developing countries 2006;2:626-8.

2 Thind A, Hsia R, Mabweijano J, et al. Prehospital and Emergency Care. In: Disease control priorities, third edition (volume 1): essential surgery, 2015: 245-62. 
3 Chang CY, Abujaber S, Reynolds TA, et al. Burden of emergency conditions and emergency care usage: new estimates from 40 countries. Emerg Med J 2016;33:794-800.

4 Leligdowicz A, Bhagwanjee S, Diaz JV, et al. Development of an intensive care unit resource assessment survey for the care of critically ill patients in resource-limited settings. J Crit Care 2017;38:172-6.

5 Baker T, Lugazia E, Eriksen J, et al. Emergency and critical care services in Tanzania: a survey of ten hospitals. BMC Health Serv Res 2013:13:140.

6 Fottrell E. Dying to count: mortality surveillance in resource-poor settings. Glob Health Action 2009;2:1926.

7 Byass P. Who needs cause-of-death data? PLoS Med 2007;4:e333.

8 Khan A, Penoff BT, Pirrotta EA, et al. Shifting the paradigm of emergency care in developing countries. Cureus 2018;10:e2219.

9 Austin S, Murthy S, Wunsch H, et al. Access to urban acute care services in high- vs. middle-income countries: an analysis of seven cities. Intensive Care Med 2014;40:342-52.

10 Hsiao M, Morris SK, Malhotra A, et al. Time-critical mortality conditions in low-income and middle-income countries. Lancet 2013;381:993-4.

11 Berthelot S, Lang ES, Quan H, et al. Identifying emergency-sensitive conditions for the calculation of an emergency care inhospital standardized mortality ratio. Ann Emerg Med 2014;63:418-24.

12 World Health Assembly. Health systems: emergency-care systems, 2007.

13 World Health Assembly. Emergency care systems for universal health coverage: ensuring timely care for the acutely ill and injured, 2019.

14 Reynolds TA, Sawe H, Rubiano AM, et al. Strengthening health systems to provide emergency care. In: Disease control priorities: improving health and reducing poverty. 3rd edn, 2017.

15 Byass P, Herbst K, Fottrell E, et al. Comparing verbal autopsy cause of death findings as determined by physician coding and probabilistic modelling: a public health analysis of 54000 deaths in Africa and Asia. J Glob Health 2015;5:010402.

16 World Health Organization. Verbal autopsy standards: the 2016 who verbal autopsy instrument, 2016

17 Byass P, Hussain-Alkhateeb L, D'Ambruoso L, et al. An integrated approach to processing WHO-2016 verbal autopsy data: the InterVA-5 model. BMC Med 2019;17:102.

18 D'Ambruoso L, Kahn K, Wagner RG, et al. Moving from medical to health systems classifications of deaths: extending verbal autopsy to collect information on the circumstances of mortality. Glob Health Res Policy 2016;1:2

19 Edem IJ, Dare AJ, Byass P, et al. External injuries, trauma and avoidable deaths in Agincourt, South Africa: a retrospective observational and qualitative study. BMJ Open 2019;9:e027576.

20 Thaddeus S, Maine D. Too far to walk: maternal mortality in context. Soc Sci Med 1994;38:1091-110.

21 Papali A, McCurdy MT, Calvello EJB. A "three delays" model for severe sepsis in resource-limited countries. J Crit Care 2015;30:861-9.

22 Musafili A, Persson Lars-Åke, Baribwira C, et al. Case review of perinatal deaths at hospitals in Kigali, Rwanda: perinatal audit with application of a three-delays analysis. BMC Pregnancy Childbirth 2017:17:85.

23 Tewari A, Pati S, Nallala S, et al. Care seeking behaviour of older adults with hip fracture in India: a qualitative study. Journal of Patient Care 2017;03.

24 Calvello EJ, Skog AP, Tenner AG, et al. Applying the lessons of maternal mortality reduction to global emergency health. Bull World Health Organ 2015;93:417-23.

25 Kahn K, Collinson MA, Gómez-Olivé FX, et al. Profile: Agincourt health and socio-demographic surveillance system. Int J Epidemiol 2012;41:988-1001.

26 African Institute for Health and Leadership Development. Minimum data sets for human resources for health and the surgical workforce in South Africa's health system, 2015.

27 Department of Health, Mpumalanga Province. Annual report for 2018/2019, 2019

28 Wallis LA, Garach SR, Kropman A. State of emergency medicine in South Africa. Int J Emerg Med 2008;1:69-71.

29 IBM. SPSS statistics. Armonk, New York: IBM, 2017.

30 QSR International. NVivo. Melbourne: QSR International, 2018.

31 World Health Organization. Injuries \& Violence: the facts, 2014.
32 Geldsetzer P, Manne-Goehler J, Bärnighausen T, et al. What research is needed to address the co-epidemics of HIV and cardiometabolic disease in sub-Saharan Africa? Lancet Diabetes Endocrinol 2018;6:7-9.

33 Basu S, Wagner RG, Sewpaul R, et al. Implications of scaling up cardiovascular disease treatment in South Africa: a microsimulation and cost-effectiveness analysis. Lancet Glob Health 2019;7:e270-80.

34 Mayosi BM, Lawn JE, van Niekerk A, et al. Health in South Africa: changes and challenges since 2009. Lancet 2012;380:2029-43.

35 Hunter JR, Chandran TM, Asmall S, et al. The ideal clinic in South Africa: progress and challenges in implementation. South African Health Review 2017:2017:111-23.

36 Audet CM, Salato J, Blevins M, et al. Educational intervention increased referrals to allopathic care by traditional healers in three high HIV-prevalence rural districts in Mozambique. PLoS One 2013;8:e70326.

37 Deprivation VF. Distance and connectivity: the adaptation of mobile phone use to a life in Wesbank, a post-Apartheid township in South Africa. Discourse, Context \& Media 2012;1:203-16.

38 Mould-Millman N-K, Rominski SD, Bogus J, et al. Barriers to accessing emergency medical services in Accra, Ghana: development of a survey instrument and initial application in Ghana. Glob Health Sci Pract 2015;3:577-90.

39 Pillai N, Foster N, Hanifa Y, et al. Patient costs incurred by people living with HIV/AIDS prior to art initiation in primary healthcare facilities in Gauteng, South Africa. PLoS One 2019;14:e0210622.

40 Groen RS, Sriram VM, Kamara TB, et al. Individual and community perceptions of surgical care in Sierra Leone. Trop Med Int Health 2014;19:107-16.

41 MacFarlane C, van Loggerenberg C, Kloeck W. International EMS systems in South Africa--past, present, and future. Resuscitation 2005;64:145-8

42 Nee-Kofi Mould-Millman C, Rominski S, Oteng R. Ambulance or TAXI? high acuity prehospital transports in the Ashanti region of Ghana. African Journal of Emergency Medicine 2014;4:8-13.

43 Dare AJ, Ng-Kamstra JS, Patra J, et al. Deaths from acute abdominal conditions and geographical access to surgical care in India: a nationally representative spatial analysis. Lancet Glob Health 2015;3:e646-53.

44 Ouma PO, Maina J, Thuranira PN, et al. Access to emergency hospital care provided by the public sector in sub-Saharan Africa in 2015: a geocoded inventory and spatial analysis. Lancet Glob Health 2018;6:e342-50.

45 Guest GD, McLeod E, Perry WRG, et al. Collecting data for global surgical indicators: a collaborative approach in the Pacific region. BMJ Glob Health 2017;2:e000376.

46 Hirose A, Borchert M, Cox J, et al. Determinants of delays in travelling to an emergency obstetric care facility in Herat Afghanistan: an analysis of cross-sectional survey data and spatial modelling. BMC Pregnancy Childbirth 2015;15:14

47 Waiswa P, Kallander K, Peterson S, et al. Using the three delays model to understand why newborn babies die in eastern Uganda. Trop Med Int Health 2010;15:964-72.

48 D'Ambruoso L, Byass P, Qomariyah SN, et al. A lost cause? extending verbal autopsy to investigate biomedical and sociocultural causes of maternal death in Burkina Faso and Indonesia. Soc Sci Med 2010;71:1728-38.

49 Roder-DeWan S, Gupta N, Kagabo DM, et al. Four delays of child mortality in Rwanda: a mixed methods analysis of verbal social autopsies. BMJ Open 2019;9:e027435.

50 Payne CF, Wade A, Kabudula CW, et al. Prevalence and correlates of frailty in an older rural African population: findings from the HAALSI cohort study. BMC Geriatr 2017:17:293.

51 Blackstock S, Witham MD, Wade AN, et al. Ability of verbal autopsy data to detect deaths due to uncontrolled hyperglycaemia: testing existing methods and development and validation of a novel weighted score. BMJ Open 2019;9:e026331.

52 Kruk ME, Gage AD, Arsenault C, et al. High-Quality health systems in the sustainable development goals era: time for a revolution. Lancet Glob Health 2018;6:e1196-252.

53 Hussain-Alkhateeb L, D'Ambruoso L, Tollman S, et al. Enhancing the value of mortality data for health systems: adding circumstances of mortality categories (COMCATs) to deaths investigated by verbal autopsy. Glob Health Action 2019;12:1680068. 